\title{
Major impact from a minor merger ${ }^{\star}$ The extraordinary hot molecular gas flow in the Eye of the NGC 4194 Medusa galaxy $\star \star$
}

\author{
S. König ${ }^{1,2}$, S. Aalto ${ }^{1}$, S. Muller ${ }^{1}$, J. S. Gallagher III ${ }^{3}$, R. J. Beswick ${ }^{4}$, E. Varenius ${ }^{1,4}$, \\ E. Jütte ${ }^{5}$, M. Krips ${ }^{2}$, and A. Adamo ${ }^{6}$
}

\footnotetext{
${ }^{1}$ Department of Space, Earth and Environment, Onsala Space Observatory, Chalmers University of Technology, 43992 Onsala, Sweden e-mail: sabine. koenig@chalmers.se

2 Institut de Radioastronomie Millimétrique, 300 Rue de la Piscine, Domaine Universitaire, 38406 Saint Martin d'Hères, France

${ }^{3}$ Department of Astronomy, University of Wisconsin, 475 N. Charter Street, Madison, WI 53706, USA

4 Jodrell Bank Centre for Astrophysics, University of Manchester, Oxford Road, Manchester, M13 9PL, UK

${ }^{5}$ Astronomisches Institut Ruhr-Universität Bochum, Universitätsstraße 150, 44780 Bochum, Germany

${ }^{6}$ Department of Astronomy, Oskar Klein Centre, AlbaNova University Centre, Stockholm University, 10691 Stockholm, Sweden
}

Received 8 December 2017 / Accepted 30 March 2018

\begin{abstract}
Context. Minor mergers are important processes contributing significantly to how galaxies evolve across the age of the Universe. Their impact on the growth of supermassive black holes and star formation is profound - about half of the star formation activity in the local Universe is the result of minor mergers.

Aims. The detailed study of dense molecular gas in galaxies provides an important test of the validity of the relation between star formation rate and HCN luminosity on different galactic scales - from whole galaxies to giant molecular clouds in their molecular gas-rich centers.

Methods. We use observations of $\mathrm{HCN}$ and $\mathrm{HCO}^{+} 1-0$ with NOEMA and of $\mathrm{CO} 3-2$ with the SMA to study the properties of the dense molecular gas in the Medusa merger (NGC 4194) at 1" resolution. In particular, we compare the distribution of these dense gas tracers with CO 2-1 high-resolution maps in the Medusa merger. To characterize gas properties, we calculate the brightness temperature ratios between the three tracers and use them in conjunction with a non-local thermodynamic equilibrium (non-LTE) radiative line transfer model.

Results. The gas represented by $\mathrm{HCN}$ and $\mathrm{HCO}^{+} 1-0$, and $\mathrm{CO} 3-2$ does not occupy the same structures as the less dense gas associated with the lower- $J$ CO emission. Interestingly, the only emission from dense gas is detected in a 200 pc region within the "Eye of the Medusa", an asymmetric $500 \mathrm{pc}$ off-nuclear concentration of molecular gas. Surprisingly, no $\mathrm{HCN}$ or $\mathrm{HCO}^{+}$is detected for the extended starburst of the Medusa merger. Additionally, there are only small amounts of $\mathrm{HCN}$ or $\mathrm{HCO}^{+}$associated with the active galactic nucleus. The CO 3-2/2-1 brightness temperature ratio inside "the Eye" is $\sim 2.5-$ the highest ratio found so far - implying optically thin $\mathrm{CO}$ emission. The $\mathrm{CO} 2-1 / \mathrm{HCN} 1-0(\sim 9.8)$ and $\mathrm{CO} 2-1 / \mathrm{HCO}^{+} 1-0(\sim 7.9)$ ratios show that the dense gas filling factor must be relatively high in the central region, consistent with the elevated $\mathrm{CO} 3-1 / 2-1$ ratio.

Conclusions. The line ratios reveal an extreme, fragmented molecular cloud population inside the Eye with large bulk temperatures $(T>300 \mathrm{~K})$ and high gas densities $\left(n\left(\mathrm{H}_{2}\right)>10^{4} \mathrm{~cm}^{-3}\right)$. This is very different from the cool, self-gravitating structures of giant molecular clouds normally found in the disks of galaxies. The Eye of the Medusa is found at an interface between a large-scale minor axis inflow and the central region of the Medusa. Hence, the extreme conditions inside the Eye may be the result of the radiative and mechanical feedback from a deeply embedded, young and massive super star cluster formed due to the gas pile-up at the intersection. Alternatively, shocks from the inflowing gas entering the central region of the Medusa may be strong enough to shock and fragment the gas. For both scenarios, however, it appears that the $\mathrm{HCN}$ and $\mathrm{HCO}^{+}$dense gas tracers are not probing star formation, but instead a post-starburst and/or shocked ISM that is too hot and fragmented to form new stars. Thus, caution is advised in taking the detection of emission from dense gas tracers as evidence of ongoing or imminent star formation.
\end{abstract}

Key words. galaxies: evolution - galaxies: individual: NGC 4194 - galaxies: starburst - galaxies: active - radio lines: ISM ISM: molecules

\footnotetext{
${ }^{\star}$ The reduced datacubes are only available available at the CDS via anonymous ftp to cdsarc.u-strasbg.fr (130.79.128.5) or via http://cdsarc.u-strasbg.fr/viz-bin/qcat?J/A+A/615/A122

$\star \star$ Based on observations carried out under project number I15AA001 with the IRAM NOEMA Interferometer. IRAM is supported by INSU/CNRS (France), MPG (Germany) and IGN (Spain).
} 


\section{Introduction}

Galaxy evolution is a fundamental part of the overall evolution of the Universe. Its sphere of influence extends from the large scales dominated bydark matter, down to the small scales ruled by dissipative baryons that can form stars and grow supermassive black holes (SMBHs; Shlosman 2013). Interactions and mergers are a known and efficient mechanism for galaxy growth (e.g., Sandage 1990, 2005; Kormendy \& Cornell 2004). Minor mergers (unequal mass progenitors, mass ratios: $\gtrsim 1: 4$ ) occur much more frequently than major mergers (equal mass progenitors, e.g., Hernquist \& Mihos 1995; Kaviraj et al. 2009; Lotz et al. 2011). Their impact on the growth of SMBHs and star formation is profound - about half of the star formation activity in the local Universe is the result of minor mergers (Kaviraj 2014, 2016).

Schmidt (1959) was the first to systematically study the connection between gas density and star formation rate (SFR) in the Milky Way. Using H $\alpha, \mathrm{CO} 1-0$ and HI, Kennicutt (1998) determined SFR surface densities and gas surface densities in a sample of normal spirals and starburst galaxies, confirming the results of Schmidt (1959). Gao \& Solomon (2004a,b) found a similar, but tighter correlation between gas density and SFR when using HCN to study the properties of dense gas in relation to star formation in luminous infrared galaxies (LIRGs), ultraluminous infrared galaxies (ULIRGs) and normal spiral galaxies.

The Medusa merger (NGC 4194, $D=39 \mathrm{Mpc}, 1^{\prime \prime}=189 \mathrm{pc}$; Beswick et al. 2005) is a minor merger that harbors a region of highly efficient star formation in its inner $2 \mathrm{kpc}-$ the star formation efficiency (SFE, $\sim 1.5 \times 10^{-8} \mathrm{yr}^{-1}$; König et al. 2014) rivals even that of well-known ULIRGs (Aalto \& Hüttemeister 2000). Low- to intermediate-density gas spans the main body of NGC 4194 - from the tidal tail at $4.7 \mathrm{kpc}$ radius, down to the central starburst (Aalto et al. 2001). High-resolution CO observations have shown that molecular gas is associated with the minor axis dust lane crossing the galaxy's main body (Aalto \& Hüttemeister 2000). The brightest CO emission is found in a striking, off-nuclear structure called the "Eye of the Medusa" (König et al. 2014). A fraction of the star formation in NGC 4194 is going on in young super star clusters (SSCs, 5-15 Myr; Bonatto et al. 1999; Pellerin \& Robert 2007) with a kpc-scale distribution (Weistrop et al. 2004; Hancock et al. 2006).

Single-dish observations revealed a global CO-to-HCN 1-0 luminosity ratio of $\sim 25$ for NGC 4194 within a $29^{\prime \prime}$ beam (Costagliola et al. 2011), a significantly higher value than for ULIRGs ( 6; e.g., Solomon et al. 1992; Curran et al. 2000), indicating that the average fraction of dense gas is significantly lower despite the similar extreme SFE.

Here we present a high-angular resolution study of the dense molecular gas in the Medusa merger using $\mathrm{HCN}, \mathrm{HCO}^{+}$and $\mathrm{CO}$ emission as tracers thereof. Throughout the paper, we are concerned with pure rotational transitions of $\mathrm{HCN}, \mathrm{HCO}^{+}, \mathrm{CO}$, $\mathrm{C}_{2} \mathrm{H}, \mathrm{SiO}, \mathrm{H}^{13} \mathrm{CO}^{+}$between upper state $J^{\prime}=j$ and lower state $J=i$ that are labeled $j-i$.

In Sect. 2 we describe the observations and how the data were reduced and analyzed, in Sect. 3 we present the results, and in Sect. 4 we discuss their implications.

\section{Observations}

\subsection{NOEMA observations}

The $\mathrm{HCN}$ and $\mathrm{HCO}^{+} 1-0$ observations of NGC 4194 were carried out with the Northern Extended Millimeter Array (NOEMA) in the frame of science verification on March 10, 2015. Data were taken with seven antennas in extended configuration, with baselines between 32 and $760 \mathrm{~m}$. Therefore, the observations are sensitive to scales smaller than $13.5^{\prime \prime}$. The phase center of the observations was located at $\alpha=12: 14: 09.660$ and $\delta=+54: 31: 35.85$ - the $1.4 \mathrm{GHz}$ radio continuum peak (J2000, Beswick et al. 2005). The $3 \mathrm{~mm}$-band receivers were tuned to $87.039 \mathrm{GHz}$ to cover the $\mathrm{HCN}$ and $\mathrm{HCO}^{+} 1-0$ lines in the $3.6 \mathrm{GHz}$ bandwidth of WideX. Also, the $\mathrm{C}_{2} \mathrm{H}, \mathrm{H}^{13} \mathrm{CO}^{+} 1-0$, and $\mathrm{SiO} 2-1$ lines were accessible in the same tuning. The instrumental spectral resolution was $1.95 \mathrm{MHz}\left(\sim 6.7 \mathrm{~km} \mathrm{~s}^{-1}\right)$. For analysis purposes we smoothed the data to $\sim 10 \mathrm{~km} \mathrm{~s}^{-1}$, resulting in $1 \sigma \mathrm{rms}$ noise levels per channel of $\sim 0.4 \mathrm{mJy} \mathrm{beam}^{-1}$ for both $\mathrm{HCN}$ and $\mathrm{HCO}^{+} 1-0$. The antenna system noise temperature $T_{\text {sys }}$ ranged from 70 to $160 \mathrm{~K}$. During the observations, different sources were observed for calibration purposes: MWC 349 and LkHa 101 as flux calibrators, 3C 84 as bandpass calibrator, and $\mathrm{J} 1150+497$ and $\mathrm{J} 1259+516$ as phase calibrators. We estimate flux calibration uncertainty of about $15-20 \%$.

Data reduction and analysis were performed using the CLIC and MAPPING software packages within GILDAS ${ }^{1}$. Applying a natural weighting scheme led to a nearly circular beam size of $\sim 1.0^{\prime \prime}$.

\subsection{SMA observations}

\subsection{1. $\mathrm{CO} 3-2$}

On April 10, 2016, additional CO3-2 observations were obtained with the Submillimeter Array (SMA) in its extended configuration with baselines between 44 and $226 \mathrm{~m}$. The $345 \mathrm{GHz}$ receivers, tuned to $342.935 \mathrm{GHz}$, were used in conjunction with the correlator in $4 \mathrm{GHz}$ mode with 128 channels and a spectral resolution of $\sim 0.8 \mathrm{MHz}\left(\sim 0.7 \mathrm{~km} \mathrm{~s}^{-1}\right)$. We smoothed the data to a velocity resolution of $\sim 10.6 \mathrm{~km} \mathrm{~s}^{-1}$, which results in a $1 \sigma \mathrm{rms}$ noise level per channel of $\sim 29.9 \mathrm{mJy} \mathrm{beam}^{-1}$. The observed data set is sensitive to scales smaller than $\sim 2.5^{\prime \prime}$ at the observing frequency. For calibration, Callisto and MWC349a (flux), J1924-292 (bandpass), and J1419+543 and J1153+495 (phase) were observed. The SMA data were calibrated using the dedicated MIR/IDL SMA reduction package. The estimated flux calibration uncertainty is of the order of $20 \%$. The visibilities were converted into FITS format and transferred to the GILDAS/MAPPING package for further imaging. Similar to the NOEMA observations, the phase center is located at $\alpha=12: 14: 09.660$ and $\delta=+54: 31: 35.85$. With natural weighting the resulting synthesized beam is $\sim 1.1^{\prime \prime} \times 1.0^{\prime \prime}$ with a position angle of $\sim 81^{\circ}$, the beam of the uniformly weighted data cube is $\sim 0.74^{\prime \prime} \times 0.66^{\prime \prime}\left(\mathrm{PA} \sim 99^{\circ}\right)$.

\subsection{2. $1 \mathrm{~mm}$ continuum}

Observations of the ${ }^{12} \mathrm{CO}$ and ${ }^{13} \mathrm{CO} 2-1$ line transitions with the SMA were taken in compact (April 08, 2010) and very extended (February 21, 2010) configuration. We extracted the $1 \mathrm{~mm}$ continuum information from these data sets. The phase center of the image presented in this work is located at $\alpha=12: 14: 09.660$ and $\delta=+54: 31: 35.85$. The resulting synthesized beam is $\sim 1.9^{\prime \prime} \times 1.8^{\prime \prime}\left(\mathrm{PA}-46^{\circ}\right)$. Imaging the compact configuration alone results in a synthesized beam of $\sim 3.4^{\prime \prime} \times 3.3^{\prime \prime}$, PA $22^{\circ}$. Baseline lengths range between 38 and $509 \mathrm{~m}$. Therefore, these data are sensitive to emission originating from scales smaller than $\sim 4.2^{\prime \prime}$ at the observing frequency of $230 \mathrm{GHz}$. We estimated calibration uncertainties on all fluxes

1 http://www.iram.fr/IRAMFR/GILDAS 

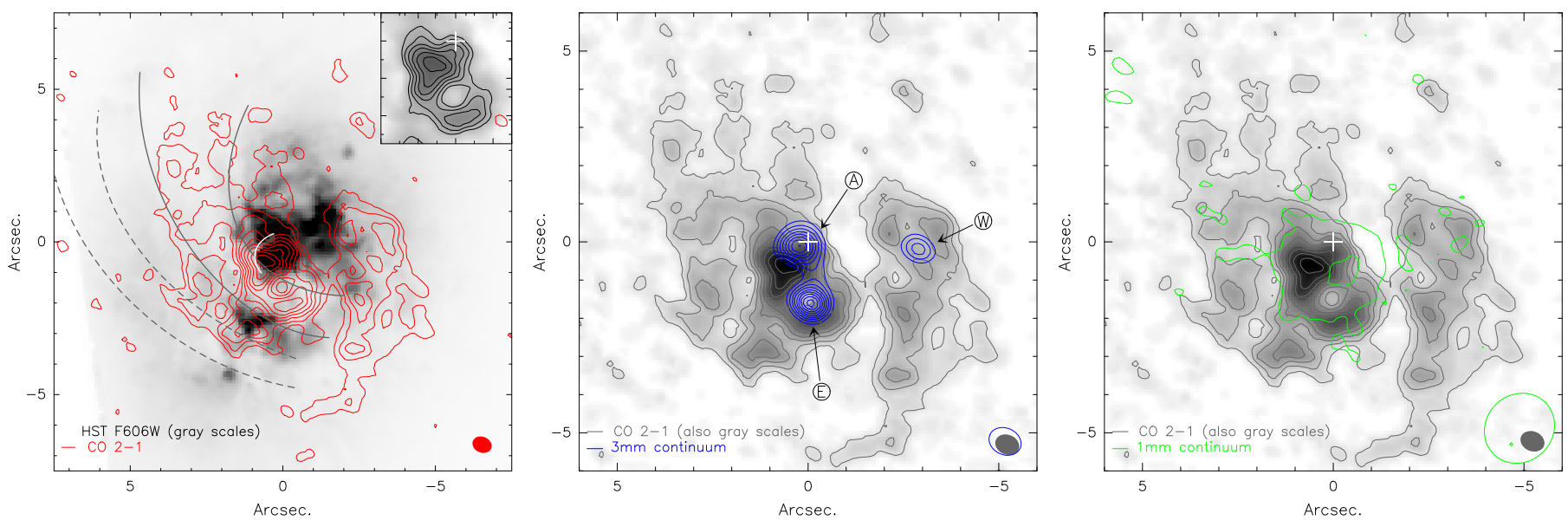

Fig. 1. Left panel: overlay of the high-resolution $\mathrm{CO} 2-1$ emission contours on top of an HST WFPC2 F606W filter image (from König et al. 2014). The insert shows a zoom into the $3^{\prime \prime} \times 3^{\prime \prime}$ surrounding the Eye of the Medusa. Contours start at $2 \sigma$ and are spaced in steps of $2 \sigma(1 \sigma$ $\sim 0.7 \mathrm{Jy}_{\text {beam }}^{-1} \mathrm{~km} \mathrm{~s}^{-1}$ ). The locations of the most important dust lanes are indicated by gray and white curves. Center panel: distribution of the integrated intensity emission of the $3 \mathrm{~mm}$ continuum (blue contours) compared to the $\mathrm{CO} 2-1$ emission (gray contours and background). The $3 \mathrm{~mm}$ continuum contours start at $5 \sigma$ and are spaced in steps of $3 \sigma\left(1 \sigma \sim 50 \mu \mathrm{Jy}\right.$ beam $\left.^{-1}\right)$. The main $3 \mathrm{~mm}$ continuum emission peak is located at the center of the Eye of the Medusa (E). Right panel: $1 \mathrm{~mm}$ continuum emission on top of the CO $2-1$ emission. Contours are at 3 and $6 \sigma(1 \sigma$ $\left.\sim 0.5 \mathrm{mJy}_{\text {beam }}{ }^{-1}\right)$. The emission peaks south of the AGN position, close to the center of the Eye. North is up, east to the left. The position of the $1.4 \mathrm{GHz}$ continuum peak is marked by a white cross (Beswick et al. 2005). Beam sizes are $0.6^{\prime \prime} \times 0.5^{\prime \prime}$ for $\mathrm{CO} 2-1,0.9^{\prime \prime} \times 0.7^{\prime \prime}$ for the $3 \mathrm{~mm}$ continuum, and $1.9^{\prime \prime} \times 1.8^{\prime \prime}$ for the $1 \mathrm{~mm}$ continuum.

of $\sim 20 \%$. For more details regarding data reduction, array combination and analysis, see König et al. (2014).

\section{Results}

\subsection{Continuum}

\subsection{1. $3 \mathrm{~mm}$ continuum}

The $3 \mathrm{~mm}$ continuum, as pictured in Fig. 1, shows three distinct, point-like sources in the center of NGC 4194 - one at the position of the active galactic nucleus (AGN, "A"), one inside the Eye ("E"), and one in the western arm ("W") of the $\mathrm{CO} 2-1$ distribution (König et al. 2014). The highest peak flux is found in the emission peak inside the Eye (see Fig. 1). The flux density recovered from the three components is $5.6 \pm 0.4 \mathrm{mJy}$ (within $3 \sigma$ contours): $2.2 \pm 0.4 \mathrm{mJy}$ for the AGN position (A), $2.0 \pm 0.4 \mathrm{mJy}$ inside the Eye (E), and $1.2 \pm 0.2 \mathrm{mJy}$ in the western arm (W).

\subsection{2. $1 \mathrm{~mm}$ continuum}

Figure 1 shows the $1 \mathrm{~mm}$ continuum distribution in the central $\sim 2 \mathrm{kpc}$ of NGC 4194. The emission peak is associated with the Eye of the Medusa, slightly south of the AGN position. The integrated intensity within $3 \sigma$ is $4.1 \pm 0.5 \mathrm{mJy}$ at a central frequency of $230.5 \mathrm{GHz}$, when combining compact and very extended configuration. Imaging only the compact configuration yields an integrated intensity of $15.5 \pm 0.5 \mathrm{mJy}$. In contrast to the $3 \mathrm{~mm}$ continuum emission, the peak associated with the Eye is of more circular shape (Figs. 1 and B.1). Also, whereas the $3 \mathrm{~mm}$ continuum shows a secondary emission peak in the western arm, the $1 \mathrm{~mm}$ continuum does not. For a side-by-side comparison of the $3 \mathrm{~mm}$ and $1 \mathrm{~mm}$ continuum emission distributions degraded to the spatial resolution of the $1 \mathrm{~mm}$ observations, see Fig. B.1.

\subsection{Line emission}

\subsection{1. $\mathrm{HCN}$ and $\mathrm{HCO}^{+} 1-0$}

The distributions of the $\mathrm{HCN}$ and $\mathrm{HCO}^{+} 1-0$ emission are quite similar to each other in NGC 4194 (Fig. 2): the main emission peak is located inside the Eye of the Medusa where CO 2-1 emission shows a distinct minimum (Fig. 1; König et al. 2014). This location is also situated inside the minor axis dust lane crossing the main body of NGC 4194. A secondary peak is located in the western arm of the $\mathrm{CO} 2-1$ distribution. A close look at the $\mathrm{HCO}^{+}$channel map also reveals low-level emission (at a $3 \sigma$ level) associated with the AGN position.

The overall $\mathrm{HCO}^{+} 1-0$ emission line is brighter than the corresponding line transition of $\mathrm{HCN}$ (cf. Fig. A.1). Measuring the intensities in the naturally weighted map with $10 \mathrm{~km} \mathrm{~s}^{-1}$ wide channels results in integrated fluxes of $1.0 \pm 0.1 \mathrm{Jy} \mathrm{km} \mathrm{s}^{-1}$ for the $\mathrm{HCO}^{+}\left(654 \pm 61 \mathrm{mJy} \mathrm{km} \mathrm{s}^{-1}\right.$ in the Eye, $374 \pm 48 \mathrm{mJy} \mathrm{km} \mathrm{s}^{-1}$ in the western arm) and $0.8 \pm 0.1 \mathrm{Jy} \mathrm{km} \mathrm{s}^{-1}$ for $\mathrm{HCN}(515 \pm$ $53 \mathrm{mJy} \mathrm{km} \mathrm{s}^{-1}$ in the Eye, $299 \pm 44 \mathrm{mJy} \mathrm{km} \mathrm{s}^{-1}$ in the western arm), respectively.

\subsection{2. $\mathrm{CO} 3-2$}

Like $\mathrm{HCN}$ and $\mathrm{HCO}^{+} 1-0, \mathrm{CO} 3-2$ has its main peak inside the Eye. Other emission regions are located in the western arm, at the AGN position and east of the AGN in the dust lane. Except for the vicinity of the AGN, these regions are all deficient in $\mathrm{CO} 2-1$ (Fig. 2). The total integrated intensity of all components combined in the lower-resolution cube amounts to $158.0 \pm 7.7 \mathrm{Jy} \mathrm{km} \mathrm{s}^{-1}\left(93.8 \pm 4.9 \mathrm{Jy} \mathrm{km} \mathrm{s}^{-1}\right.$ in the Eye, and $39.5 \pm 3.4 \mathrm{Jy} \mathrm{km} \mathrm{s}^{-1}$ in the western arm).

\subsubsection{Additional emission lines}

Other relevant emission lines in the $3.6 \mathrm{GHz}$ wide band are $\mathrm{C}_{2} \mathrm{H} N=1-0, \mathrm{H}^{13} \mathrm{CO}^{+} J=1-0, \mathrm{SiO} J=2-1 . \mathrm{C}_{2} \mathrm{H}$ has been detected with an integrated flux of $350 \pm 103 \mathrm{mJy} \mathrm{km} \mathrm{s}^{-1}$ (see Fig. A.1). Due to the non-detection of $\mathrm{H}^{13} \mathrm{CO}^{+} 1-0$ and $\mathrm{SiO} 2-1$, only upper limits of $\sim 2.4 \mathrm{mJy} \mathrm{km} \mathrm{s}^{-1}$ and $\sim 2.5 \mathrm{mJy} \mathrm{km} \mathrm{s}^{-1}$, respectively, are available for the fluxes of these line transitions. The limits in the integrated fluxes have been obtained from an area corresponding to the largest extension of the $\mathrm{HCO}^{+}$ emission, and within a velocity range of $\pm 85 \mathrm{~km} \mathrm{~s}^{-1}$ of the respective line centers. 

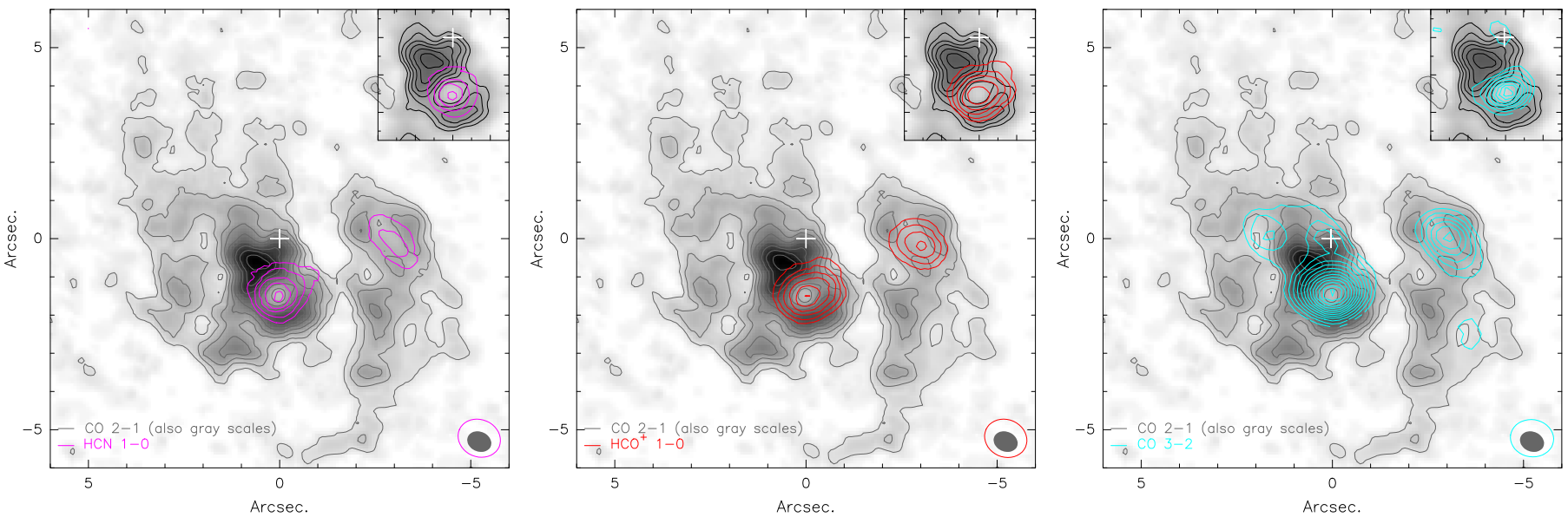

Fig. 2. Integrated intensity distributions of the $\mathrm{HCN} 1-0$ (left panel, in magenta), $\mathrm{HCO}^{+} 1-0$ (centre panel, in red) and $\mathrm{CO} 3-2$ emission contours (right panel, in light blue) at the same spatial resolution $\left(\sim 1.1^{\prime \prime} \times 1.0^{\prime \prime}\right)$ on top of the CO $2-1$ high-resolution emission (background and

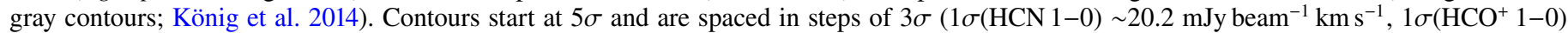
$\left.\sim 21.2 \mathrm{mJy}_{\text {beam }}{ }^{-1} \mathrm{~km} \mathrm{~s}^{-1}, 1 \sigma(\mathrm{CO} 3-2) \sim 1.6 \mathrm{Jy} \mathrm{beam}^{-1} \mathrm{~km} \mathrm{~s}^{-1}\right)$. The inset in each of the images shows the higher-resolution data for each tracer (beam sizes: $1.0^{\prime \prime} \times 0.8^{\prime \prime}, 1.0^{\prime \prime} \times 0.8^{\prime \prime}, 0.7^{\prime \prime} \times 0.7^{\prime \prime}$ ). North is up, east to the left. The position of the $1.4 \mathrm{GHz}$ continuum peak is marked by a white cross (Beswick et al. 2005).

Table 1. Characterizing properties of the interferometric observations.

\begin{tabular}{lcccccc}
\hline \hline Line & $\begin{array}{c}v_{\text {obs }} \\
{[\mathrm{GHz}]}\end{array}$ & Observatory & Configuration & $\begin{array}{c}B_{\min }{ }^{a} \\
{[\mathrm{~m}]}\end{array}$ & $\begin{array}{c}B_{\max }{ }^{b} \\
{[\mathrm{~m}]}\end{array}$ & $\begin{array}{c}\Theta_{\mathrm{MRS}}{ }^{c} \\
{[\operatorname{arcsec}]}\end{array}$ \\
\hline $\mathrm{CO} 3-2$ & 342.935 & SMA & Extended & 44 & 226 & 4.1 \\
$\mathrm{CO}^{2}-1$ & 228.631 & SMA & Compact + Very Extended & 38 & 509 & 7.1 \\
$\mathrm{HCO}^{+} 1-0$ & 88.451 & NOEMA & 7A-Special & 32 & 760 & 21.8 \\
$\mathrm{HCN} 1-0^{-87.899}$ & NOEMA & 7A-Special & 32 & 760 & 22.0 \\
\hline
\end{tabular}

Notes. ${ }^{(a)}$ Shortest baseline in the data set. ${ }^{(b)}$ Longest baseline in the data set. ${ }^{(c)}$ Maximum recoverable scale. The interferometer in the given configuration is sensitive to structures smaller than this size scale.

\subsection{Interferometric line ratios}

The determination of molecular line ratios is a very powerful method to explore the physical and chemical processes in the interstellar medium. However, one has to be aware of possible implications when using interferometric data. Indeed, an interferometer is sensitive to a limited range of spatial scales. Extended structures might be filtered out if the shortest array baselines are still too long. When comparing two lines, one must therefore ensure that the corresponding data are sampling similar spatial scales. Table 1 gives an overview of the properties for each observational setup. $\Theta_{\mathrm{MRS}}$, the maximum recoverable scale, is defined as $\Theta_{\mathrm{MRS}} \approx 0.6 \times \lambda / B_{\min }$ (e.g., the ALMA Cycle 5 Technical Handbook), where $B_{\min }$ is the shortest projected baseline length. For our observations $\Theta_{\mathrm{MRS}}$ ranges between $4.1^{\prime \prime}$ for CO3-2 and 22.0" for HCN 1-0, i.e., significantly larger than the dip of emission in $\mathrm{CO} 2-1$ in the Eye. Additionally, we smooth each data set to the same beam size $\left(1.0^{\prime \prime} \times 1.0^{\prime \prime}\right)$ for the line ratio determination, and we extract the integrated intensities from within the same region.

The $\mathcal{R}_{32 / 21}=\mathrm{CO} 3-2 / \mathrm{CO} 2-1$ brightness temperature ratio is large, $\sim 2.5$, at position $\mathrm{E}$, inside the Eye of the Medusa (see Table 2). The ratios were determined inside a circular $1^{\prime \prime}$ polygon. This is slightly larger than the area inside the Eye where CO 2-1 is deficient. Thus, it is very likely that the inner walls of the Eye of the Medusa contribute to the measured CO 2-1 flux inside the polygon. Hence, the $\mathrm{CO} 3-2 / \mathrm{CO} 2-1$ ratio we determined is a lower limit to $\mathcal{R}_{32 / 21}$ inside the Eye. To measure $\mathcal{R}_{32 / 21}$ more precisely, higher-resolution observations are needed.

\section{Discussion}

\subsection{Dense gas distribution}

A strong correlation between SFR and integrated molecular line luminosity from dense gas is seen in most galaxies (e.g., Gao \& Solomon 2004a,b). For the Medusa this would imply that due to its high SFR (FIR: 6-7 $M_{\odot} \mathrm{yr}^{-1}-$ Aalto \& Hüttemeister 2000; $\mathrm{H} \alpha: \sim 10 M_{\odot} \mathrm{yr}^{-1}$ - Gallagher, in prep.) the HCN luminosity should be high, however, this is not the case: On global galaxy scales, single-dish observations revealed a low $\mathrm{HCN}$ to-CO 1-0 luminosity ratio similar to values found for GMCs in the galactic disks of M 31 and the Milky Way (Brouillet et al. 2005; Costagliola et al. 2011; Matsushita et al. 2015), indicating that the fraction of dense gas should be significantly lower than in ULIRGs despite the similarly extreme SFE. However, our high-angular-resolution observations paint a different picture: the dense gas is not distributed throughout the starburst region in the central $2 \mathrm{kpc}$ of the Medusa (e.g., Armus et al. 1990; Wynn-Williams \& Becklin 1993; Prestwich et al. 1994; Aalto \& Hüttemeister 2000). Instead, it is located in a compact region inside the Eye of the Medusa where the $\mathrm{CO} 2-1$ emission shows a distinct minimum (Fig. 2), thus explaining the relative 
Table 2. Brightness temperatures and line widths in the Eye of the Medusa and the western arm.

\begin{tabular}{lcccccc}
\hline \hline Line & \multicolumn{3}{c}{ Eye of the Medusa } & \multicolumn{3}{c}{ Western arm } \\
& $\begin{array}{c}\int T_{\mathrm{B}} \mathrm{d}^{a, b} \\
{\left[\mathrm{~K} \mathrm{~km} \mathrm{~s}^{-1} \mathrm{pc}^{2}\right]}\end{array}$ & $\begin{array}{c}\text { peak } T_{\mathrm{B}}{ }^{a, c} \\
{[\mathrm{~K}]}\end{array}$ & $\begin{array}{c}\mathrm{FWHM}^{d} \\
{\left[\mathrm{~km} \mathrm{~s}^{-1}\right]}\end{array}$ & $\begin{array}{c}\int T_{\mathrm{B}} \mathrm{d} v^{a, b} \\
{\left[\mathrm{~K} \mathrm{~km} \mathrm{~s}^{-1} \mathrm{pc}^{2}\right]}\end{array}$ & $\begin{array}{c}\text { peak } T_{\mathrm{B}}{ }^{a, c} \\
{[\mathrm{~K}]}\end{array}$ & $\begin{array}{c}\mathrm{FWHM}^{d} \\
{\left[\mathrm{~km} \mathrm{~s}^{-1}\right]}\end{array}$ \\
\hline $\mathrm{CO} 3-2$ & $892.3 \pm 6.3$ & $13.82 \pm 0.42$ & $74.7 \pm 3.1$ & $504.4 \pm 3.3$ & $7.48 \pm 0.27$ & $66.2 \pm 2.7$ \\
$\mathrm{CO} 2-1$ & $356.8 \pm 0.6$ & $3.74 \pm 0.08$ & $88.8 \pm 2.0$ & $208.4 \pm 0.4$ & $2.01 \pm 0.06$ & $91.7 \pm 2.9$ \\
$\mathrm{HCO}^{+} 1-0$ & $45.3 \pm 0.5$ & $0.80 \pm 0.03$ & $63.7 \pm 3.3$ & $35.9 \pm 0.3$ & $0.62 \pm 0.03$ & $55.7 \pm 2.9$ \\
$\mathrm{HCN} 1-0$ & $36.4 \pm 0.2$ & $0.53 \pm 0.03$ & $59.6 \pm 4.2$ & $23.7 \pm 0.3$ & $0.51 \pm 0.04$ & $63.6 \pm 8.6$ \\
\hline
\end{tabular}

Notes. ${ }^{(a)}$ The brightness temperatures $T_{\mathrm{B}}$ were determined from resolution-matched $\left(1^{\prime \prime}\right)$ data cubes inside the same polygons of $1^{\prime \prime}$ size. ${ }^{(b)} \int T_{\mathrm{B}} \mathrm{d} v$ is the brightness temperature integrated over the $1^{\prime \prime}$ polygon. ${ }^{(c)}$ peak $T_{\mathrm{B}}$ is the peak brightness temperature of the spectrum integrated over the $1^{\prime \prime}$ polygon. ${ }^{(d)}$ Obtained from a single Gaussian fit to the line.

faintness on global scales. A comparison between the $\mathrm{CO} 2-1$ emission and the young superstar clusters (SSCs) in the Medusa has shown that many young massive clusters are located away from the bulk of the CO (Weistrop et al. 2004; Hancock et al. 2006; König et al. 2014) - the same effect is visible for the dense gas, only in a more extreme fashion. So far, only one cluster has been identified in the vicinity of the Eye (Hancock et al. 2006). However, the relative astrometric uncertainty between the optical and millimeter data preclude a definite conclusion.

An in-detail kinematic comparison between the $\mathrm{HCN}$, $\mathrm{HCO}^{+} 1-0$ and $\mathrm{CO} 3-2$, and the $\mathrm{CO} 2-1$ emission in the Eye of the Medusa shows strong evidence for their different origins. A CO 2-1 spectrum obtained from the center of the Eye shows a full width at half maximum (FWHM) line width of $\sim 90 \mathrm{~km} \mathrm{~s}^{-1}$. The $\mathrm{HCN}$ and $\mathrm{HCO}^{+} 1-0$ spectra obtained from within the same area instead show a FWHM line width of $\sim 60 \mathrm{~km} \mathrm{~s}^{-1}$ (see Table 2). The centroid velocities of the lines, however, do not differ significantly (i.e., by $\lesssim 10 \mathrm{~km} \mathrm{~s}^{-1}$ ). This may suggest that $\mathrm{CO} 2-1$ is tracing a more extended, lower-density component. The CO 2-1 intensity at high velocities is roughly the same in the northern and southern parts of the Eye, while at lower velocities it is strongly dominated by emission in the North. The dense gas emission does not show such a behavior - their centroids do not move at all. Thus, the evidence presented here points toward different origins of the dense gas and the $\mathrm{CO} 2-1$ emission.

\subsection{Origin and physical properties of the dense gas emission in the Eye}

\subsubsection{Excitation and line ratios}

Previous studies, using single-dish as well as interferometric observations, mark the $\mathcal{R}_{32 / 21}$ we observed for the Eye $\left(\mathcal{R}_{32 / 21} \sim 2.5\right.$; see Sect. 3.3) as an extreme case; observations of a large number of luminous infrared galaxies show that values of $\mathcal{R}_{32 / 21}$ greater than unity are rare. So far the highest $\mathcal{R}_{32 / 21}$ found are at $\sim 1.9$ (e.g., Greve et al. 2009; Papadopoulos et al. 2012). Interferometric observations show that even more powerful ULIRGs, such as, for example, Arp 220 and NGC 6240, show $\mathcal{R}_{32 / 21} \lesssim 1$ (e.g., Greve et al. 2009; Sliwa \& Downes 2017). Thus, ratios measured in the Eye of the Medusa indicate truly exceptional properties of the dense gas.

$\mathcal{R}_{32 / 21}$ together with brightness temperature ratios of $\mathrm{CO} 3-2$ with $\mathrm{HCN} 1-0$ and $\mathrm{HCO}^{+} 1-0$ (Table 2) can help us to infer the gas properties in this extraordinary region. We perform a line excitation analysis using the radiative transfer code RADEX (van der Tak et al. 2007) by exploring a grid of parameters in kinetic temperature $(50-500 \quad \mathrm{~K})$ and $\mathrm{H}_{2}$ density $\left(10^{3}-10^{6} \mathrm{~cm}^{-3}\right)$ for a $\mathrm{CO}$ column density $(\mathrm{N}(\mathrm{CO}) / \Delta \mathrm{v})$ between $10^{16}$ and $10^{18} \mathrm{~cm}^{-2}$. Figure 3 shows the results of the radiative transfer modeling: high values $(>2.2)$ of $\mathcal{R}_{32 / 21}$ can only be achieved for warm gas $(T>300 \mathrm{~K}), \mathrm{H}_{2}$ densities of a few $10^{4} \mathrm{~cm}^{-3}$ and $\mathrm{CO}$ column densities of $10^{16} \mathrm{~cm}^{-2}$. Assuming that $\mathrm{HCN}, \mathrm{HCO}^{+} 1-0$ and $\mathrm{CO} 3-2$ trace the same gas component, that is, the same physical conditions in the gas, we tune the relative abundance ratios for these three molecules to match the line ratios for a given density. Adopting also similar beam-filling factors for each tracer, we estimate relative abundances for $\mathrm{CO} / \mathrm{HCN}$ of $\sim 4000$ and for $\mathrm{CO} / \mathrm{HCO}^{+}$of $\sim 9000$. However, since we only have two $\mathrm{CO}$ transitions (2-1 and 3-2) and one transition (1-0) each of $\mathrm{HCN}$ and $\mathrm{HCO}^{+}$, the range of possible $\mathrm{H}_{2}$ gas densities is not very well constrained. To change this, observations of additional transitions of $\mathrm{HCN}$ and/or $\mathrm{HCO}^{+}$are necessary.

\subsubsection{The nature of the dense gas clouds}

As a first step and the simplest scenario, we assume that the dense gas emission originates from one large virialized cloud with a radius of $\sim 1^{\prime \prime}$ with $n\left(\mathrm{H}_{2}\right) \geq 5 \times 10^{3} \mathrm{~cm}^{-3}$ and $T_{k} \sim 300 \mathrm{~K}$. This results in an expected line width (FWHM) of the emission line of about $540 \mathrm{~km} \mathrm{~s}^{-1}$. The observed line widths for all four lines, however, are less than $\sim 100 \mathrm{~km} \mathrm{~s}^{-1}$ inside the Eye (see Table 2). Furthermore, such a structure would have a gas mass of about $10^{10} M_{\odot}$ which is larger than the gas mass of most galaxies. Thus, the single-cloud, high-filling-factor scenario is unrealistic. Alternatively, it is possible that the emission is coming from a larger number of small, self-gravitating dense gas clumps. Assuming a typical clump size of $5 \mathrm{pc}$ instead, the scale of dense molecular clouds (see e.g., Lizano \& Shu 1987; Nguyen Luong et al. 2011) results in line widths of about $10 \mathrm{~km} \mathrm{~s}^{-1}$. This scenario is a possibility if the filling factor in the gas is high. As a result of this exercise, it seems that the dense gas in the Eye of the Medusa is most likely fragmented into a large number of small filaments or clouds.

\subsection{What is inside the Eye of the Medusa?}

\subsubsection{Spectral index}

Continuum observations in the radio and millimeter regimes can provide information concerning the excitation mechanism for the dense gas emission. For the Medusa, high-resolution continuum data at $150 \mathrm{MHz}$ (LOFAR; König et al., in prep.), 1.4, 5, 8.4, 15, and $22 \mathrm{GHz}$ (eMERLIN, VLA; Beck et al. 2014; König et al. in prep.), and at 86, 230 and $345 \mathrm{GHz}$ (this work, König et al. 2014) 

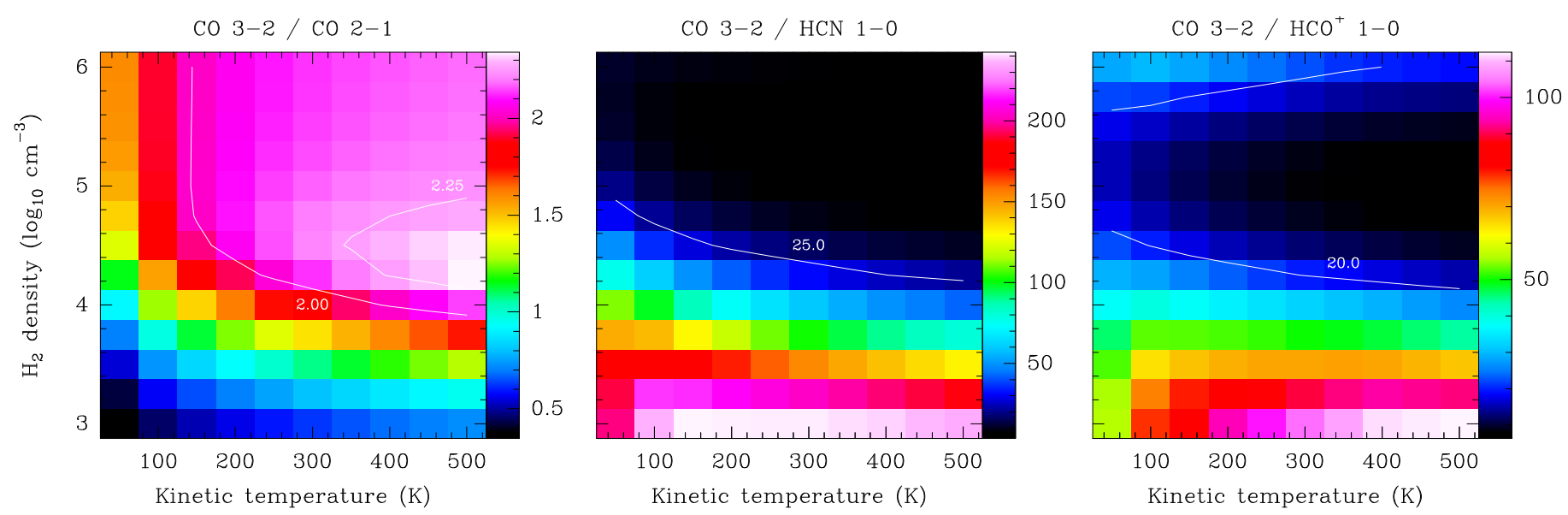

Fig. 3. Results of the RADEX analysis of the $\mathrm{CO} 3-2 / \mathrm{CO} 2-1, \mathrm{CO} 3-2 / \mathrm{HCN} 1-0$, and $\mathrm{CO} 3-2 / \mathrm{HCO}^{+} 1-0$ brightness temperature ratios. The white curves and associated numbers indicate $T_{\mathrm{B}}$ ratios toward the Eye of the Medusa of 2 and 2.25 for CO 3-2/CO 2-1, 25 for CO $3-2 / \mathrm{HCN} 1-0$, and 20 for $\mathrm{CO} 3-2 / \mathrm{HCO}^{+} 1-0$, respectively. The range in $\mathrm{H}_{2}$ densities and kinetic temperatures are the same for each of the three plots. The $\mathrm{CO}$ column density $(\mathrm{N}(\mathrm{CO}) / \Delta \mathrm{v})$ is $10^{16} \mathrm{~cm}^{-2}$. The color scheme indicates possible brightness temperature ratios for each combination of tracers.

show spectral indices indicative of a mixture of non-thermal synchrotron and thermal free-free emission. These emission processes are usually associated with supernovae ( $\mathrm{SNe}$ ) and AGN activity (synchrotron) and young star formation (free-free; e.g., Beck et al. 2014; Schober et al. 2017, and references therein).

In an earlier paper, we showed that it is possible that the energetic output from a large number of supernova explosions could have led to the observed shell-like morphology of the CO 2-1 emission in the Eye of the Medusa (König et al. 2014). Far-infrared (FIR) and X-ray observations hint towards the presence of low-level AGN activity at the dynamic center of the Medusa (e.g., Kaaret \& Alonso-Herrero 2008; Bernard-Salas et al. 2009; Lehmer et al. 2010). The spatially resolved studies (Kaaret \& Alonso-Herrero 2008; Lehmer et al. 2010) found the activity to be associated with the main $1.4 \mathrm{GHz}$ radio continuum emission peak north of the Eye (Beswick et al. 2005). So far no indications for an AGN inside the Eye of the Medusa have been found. Thus, supernovae are the most likely contributor of the synchrotron emission in the Eye.

The free-free component contributing to the continuum emission could be due to the presence of young star clusters inside the Eye, which could be heavily obscured (Hancock et al. 2006). However, the faint millimeter continuum emission towards higher frequencies for the Eye (see Sect.3.1) indicates that there is very little cold dust emission associated with the dense gas in this region. The high gas temperature (see Sect. 4.2.1) most likely leads to the dust being heated and thus its emission peak is shifted to shorter thermal infrared wavelengths. Observations at these wavelengths, that would provide a test of this hypothesis, are currently not available for the Medusa.

The mixed spectral index indicates that the age of the star formation in the Eye would be at least a few $10^{6} \mathrm{yr}$, since it is at this stage in massive stellar evolution that the first supernovae occur.

\subsection{2. $\mathrm{HCN}$ vs. $\mathrm{HCO}^{+}$}

Single-dish observations of the total molecular gas and the dense molecular gas on large scales have shown differences in the global properties in a range of activity types in galaxies (e.g., Solomon et al. 1997; Graciá-Carpio et al. 2008; Krips et al. 2008; Privon et al. 2015). Several studies have found intrinsic differences between the way dense molecular gas content is traced by, for example, $\mathrm{HCN}$ and $\mathrm{HCO}^{+}$, and the distribution of low$J$ CO transitions as tracers of the total gas properties: $\mathcal{R}_{\mathrm{HCN} / \mathrm{CO}}$, the HCN-to-CO ratio, is small in quiescent and starburst galaxies $(<0.3$, Gao \& Solomon 2004b; Krips et al. 2007; Matsushita et al. 2015, and references therein) compared to galaxies with AGN activity (up to 1 or more, Usero et al. 2004; Krips et al. 2007; Matsushita et al. 2015, and references therein). On small scales, $\mathcal{R}_{\mathrm{HCN} / \mathrm{CO}}$ can be significantly different from values found on galaxy scales (e.g., in NGC 1068, NGC 6951, and M 51; Kohno et al. 1996; Krips et al. 2007, 2011; Matsushita et al. 2015). Our high-resolution ( $\left.1^{\prime \prime}\right) \mathrm{HCN} 1-0$-to-CO 2-1 line ratio is $\sim 0.20$ in the Eye of the Medusa. An interferometric CO 2-1to-CO 1-0 line ratio map yields values of $\sim 0.9$ in the Eye (König et al., in prep.). Taking the $\mathrm{CO}$ ratio into account, the HCN 1-0to-CO 2-1 ratio translates to $\mathrm{HCN} 1-0$-to-CO 1-0 of $\sim 0.1$. This places the Eye in a regime close to what has been found for LIRGs and ULIRGs (e.g., Gao et al. 2007; Usero et al. 2015).

It has also been shown that $\mathcal{R}_{\mathrm{HCN} / \mathrm{HCO}^{+}}$, the $\mathrm{HCN}$-to- $\mathrm{HCO}^{+}$ line ratio, can be significantly higher close to AGN (nuclear) compared to regions dominated by starbursts, and regions of more quiescent star formation (e.g., Graciá-Carpio et al. 2006, 2008; Krips et al. 2008; Kohno et al. 2008; Davies et al. 2012), though it can also be high in young (pre-synchrotron) star forming regions. $\mathcal{R}_{\mathrm{HCN} / \mathrm{HCO}^{+}}$inside the Eye is approximately 0.9, which would place the Eye of the Medusa in the regime of starburst galaxies.

\subsection{3. $\mathrm{CN}$ as a tracer of radiative feedback}

The reasons for the enhancement of $\mathrm{HCN}$ and/or $\mathrm{HCO}^{+}$relative to $\mathrm{CO}$ and/or each other are diverse: strong UV/X-ray radiation fields (e.g., Usero et al. 2004; Meijerink et al. 2006, 2007; Krips et al. 2007, 2011; Matsushita et al. 2015), energetic particles from AGN and/or jets, cosmic rays (e.g., Meijerink et al. 2006; Bayet et al. 2010, and references therein), young star formation (e.g., Pirogov 1999; Boonman et al. 2001; Lahuis et al. 2007, and references therein), IR-pumping of HCN (e.g., Carroll \& Goldsmith 1981; Aalto et al. 1995; Matsushita et al. 2015), and high-temperature gas chemistry. A molecule that can help differentiate between these scenarios is $\mathrm{CN}$. This molecule can be formed from $\mathrm{HCN}$ as a result of intense UV radiation in photodissociation regions (PDRs; e.g., Aalto et al. 2002; Baan et al. 2008; Han et al. 2015). Costagliola et al. (2011) found that in 
the Medusa, globally, CN $1-0$ is found at higher intensity than $\mathrm{HCN} 1-0$. If we assume that the $\mathrm{CN}$ and $\mathrm{HCN}$ emission originate from the same spatial region inside the Eye of the Medusa, the $\mathrm{CN}$-to-HCN $1-0$ brightness temperature ratio is $\sim 2.1$. This might imply that a large part of the $\mathrm{HCN}$ was photodissociated into $\mathrm{CN}$ due to the intense $\mathrm{UV}$ radiation from nearby young massive stars.

\subsubsection{Scenarios}

What we know so far about the properties of the central ISM in the Medusa is that the gas inside the Eye is hot and dense, that there is very little cold dust, and that a mixture of synchrotron and free-free emission processes take place in this region. What exactly is going on in this region is not yet clearly determined. So far, the evidence seems to point towards post-burst radiative feedback from young massive stars as the energetic source of the observed phenomena. In this scenario, $\mathrm{HCN}$ and $\mathrm{HCO}^{+} 1-0$, and CO 3-2 are enhanced in the dense, hot gas that is associated with deeply embedded, fragmenting star-formation cores.

Alternatively however, the dense gas properties could also be the immediate result of shocks, in which the gas is compressed and heated. CO studies have shown that shock-excited molecular gas can reach temperatures and densities similar to, or above, the values we find for the Eye of the Medusa (e.g., NGC 1068, NGC 6240, NGC 7130; Hailey-Dunsheath et al. 2012; Meijerink et al. 2013; Pozzi et al. 2017).

To be able to distinguish between the two scenarios and better determine the small-scale structure of the dense gas in the Eye, further observations are necessary. Higher-resolution data sets of dense gas tracers, like the ones discussed in this paper, will help determine the degree of fragmentation in the Eye ISM, and to track the locations of shocks and/or clouds in which the massive stars are embedded. Observations of tracers such as $\mathrm{SiO}, \mathrm{CH}_{3} \mathrm{OH}$ at millimeter wavelengths, and $\mathrm{NIR} \mathrm{H}_{2}$ will directly reveal the presence of shocks, whereas $\mathrm{CN}$ observations will reveal UV-photon dominated regions close to stars. Also, shocks do not heat dust as effectively as they heat gas (e.g., Meijerink et al. 2013). Therefore, a good test will be to determine the conditions in the warm dust at infrared wavelengths.

\subsection{The importance of interaction-induced gas flows}

Studies have shown that the global CO-to-HCN ratio does not necessarily reflect on the small-scale properties of the dense gas, that is, a global value is not representative of the small-scale processes leading to the observed ratios (e.g., in NGC 1068, NGC 6951, and M 51; Kohno et al. 1996; Krips et al. 2007, 2011). For the Medusa, its relatively high global CO-to-HCN ratio implies $\mathrm{HCN}$, and thus the dense gas, to be faint. This is puzzling however, especially when taking into account the Medusa's high SFE. Following Kennicutt (1998) and Gao \& Solomon (2004a,b), one would expect the known starforming complexes to be associated with dense molecular gas. Our high-resolution observations show, however, that the dense gas is located in a very compact configuration inside the Eye of the Medusa, away from most of the optically visible ongoing star formation and the bulk of the molecular gas. However, the $3 \mathrm{~mm}$ continuum associated with the dense gas inside the Eye yields an SFR of about $2.8 M_{\odot} \mathrm{yr}^{-1}$ (following Murphy et al. 2011). Therefore, obscured star formation seems to be on-going in this region. An important factor contributing to this puzzle could be the presence of inflowing gas in this minor merger.
It has been shown that the inflow of molecular gas in galaxy mergers or interactions can lead to an increase in and/or the triggering of star formation (e.g., Meier et al. 2002; Whitmore 2007; Turner et al. 2015, and references therein). The Medusa is suspected to harbor a molecular gas inflow via its kpc-scale dust lane to the nucleus (Aalto et al. 2010; König et al. 2014), similar to what has been found in, for example, NGC 5253 (Turner et al. 2015). It has been suggested that NGC 5253 has experienced an encounter with another galaxy in its history. Observations of the dense molecular gas traced by $\mathrm{CO} 3-2$ in this dwarf galaxy led the authors to conclude that the increased star formation activity they find in a gas cloud previously only weakly detected in CO 2-1 is the result of gas inflow. Molecular gas is being transported along the minor axis dust lane, east of the nucleus, towards the galaxy center (the "streamer"). Associated with the CO3-2-detection is an "extremely" dusty gas cloud that contains two very young stellar clusters of a few times $10^{5} M_{\odot}$ each (Calzetti et al. 2015). The star formation inside these clusters was likely triggered as a result of the interaction of the inflowing gas with the prevailing ISM (Turner et al. 2015).

This scenario is a strong possibility for NGC 4194 as well. However, the affected region in the Eye of the Medusa $(r \sim 185 \mathrm{pc}$ ) is much larger (about 100 times the area) than what has been found in NGC $5253(\sim 31 \mathrm{pc} \times 11 \mathrm{pc})-$ enough space to host several star clusters. Also, in contrast to NGC 5253, very little cold dust emission is associated with the dense gas in the Eye (see Sect.4.3.1). Furthermore, only a small amount of the dense gas emission in the Medusa is found in the dust lane associated with the gas inflow (see Figs. 1 and 2). Whether these differences could be due to the dissimilar size scales in NGC 5253 and the Medusa, or a difference in the time scales of the processes induced by the gas inflow, is unclear.

\section{Conclusions}

In this paper, we studied the distribution and properties of the dense gas in the Medusa merger through observations of $\mathrm{HCN}$ and $\mathrm{HCO}^{+} 1-0$ and $\mathrm{CO} 3-2$. Surprisingly, all these tracers are located inside the Eye of the Medusa, where CO 2-1 emission shows a distinct minimum. The gas inside the Eye is hot $(>300 \mathrm{~K})$ and dense $\left(\gtrsim 10^{4} \mathrm{~cm}^{-3}\right)$. We propose two possible scenarios to explain what could cause the extreme properties of the gas inside the Eye of the Medusa: 1) They are caused by shocks as the gas flow, that transports molecular gas via the dust lane to the center of the Medusa, collides with the central ISM inside the Eye, or 2) they are an effect of supernova-induced shocks and/or radiative feedback from embedded massive star clusters, where the star formation is fed by the gas flow. Additional higher-resolution observations $\left(\sim 0.5^{\prime \prime}\right)$ of, for example, $\mathrm{HCN}, \mathrm{HCO}^{+} 3-2$, and $\mathrm{CN} 2-1$, as well as $\mathrm{H}_{2}$ NIR observations will help us disentangle the energetic processes in the Eye of the Medusa to determine what caused the extreme properties in the dense gas.

Acknowledgements. We thank the referee for useful comments. A.A. acknowledges the support of the Swedish Research Council (Vetenskapsrådet) and the Swedish National Space Board (SNSB). The Submillimeter Array is a joint project between the Smithsonian Astrophysical Observatory and the Academia Sinica Institute of Astronomy and Astrophysics and is funded by the Smithsonian Institution and the Academia Sinica. This paper is based in part on data obtained with the International LOFAR Telescope (ILT) under project code LC7_006. LOFAR (van Haarlem et al. 2013) is the Low Frequency Array designed and constructed by ASTRON. It has observing, data processing, and data storage facilities in several countries, that are owned by various parties (each with their own funding sources), and that are collectively operated by the ILT foundation 
under a joint scientific policy. The ILT resources have benefitted from the following recent major funding sources: CNRS-INSU, Observatoire de Paris and Université d'Orléans, France; BMBF, MIWF-NRW, MPG, Germany; Science Foundation Ireland (SFI), Department of Business, Enterprise and Innovation (DBEI), Ireland; NWO, The Netherlands; The Science and Technology Facilities Council, UK. MERLIN/eMERLIN is a National Facility operated by the University of Manchester at Jodrell Bank Observatory on behalf of STFC. This research has made use of the NASA/IPAC Extragalactic Database (NED) which is operated by the Jet Propulsion Laboratory, California Institute of Technology, under contract with the National Aeronautics and Space Administration.

\section{References}

Aalto, S., \& Hüttemeister, S. 2000, A\&A, 362, 42

Aalto, S., Booth, R. S., Black, J. H., \& Johansson, L. E. B. 1995, A\&A, 300, 369

Aalto, S., Hüttemeister, S., \& Polatidis, A. G. 2001, A\&A, 372, L29

Aalto, S., Polatidis, A. G., Hüttemeister, S., \& Curran, S. J. 2002, A\&A, 381, 783

Aalto, S., Beswick, R., \& Jütte, E. 2010, A\&A, 522, A59

Armus, L., Heckman, T. M., \& Miley, G. K. 1990, ApJ, 364, 471

Baan, W. A., Henkel, C., Loenen, A. F., Baudry, A., \& Wiklind, T. 2008, A\&A 477,747

Bayet, E., Hartquist, T. W., Viti, S., Williams, D. A., \& Bell, T. A. 2010, A\&A, 521, A16

Beck, S. C., Lacy, J., Turner, J., Greathouse, T., \& Neff, S. 2014, ApJ, 787, 85

Bernard-Salas, J., Spoon, H. W. W., Charmandaris, V., et al. 2009, ApJS, 184, 230

Beswick, R. J., Aalto, S., Pedlar, A., \& Hüttemeister, S. 2005, A\&A, 444, 791

Bonatto, C., Bica, E., Pastoriza, M. G., \& Alloin, D. 1999, A\&A, 343, 100

Boonman, A. M. S., Stark, R., van der Tak, F. F. S., et al. 2001, ApJ, 553, L63

Brouillet, N., Muller, S., Herpin, F., Braine, J., \& Jacq, T. 2005, A\&A, 429, 153

Calzetti, D., Johnson, K. E., Adamo, A., et al. 2015, ApJ, 811, 75

Carroll, T. J., \& Goldsmith, P. F. 1981, ApJ, 245, 891

Costagliola, F., Aalto, S., Rodriguez, M. I., et al. 2011, A\&A, 528, A30

Curran, S. J., Aalto, S., \& Booth, R. S. 2000, A\&AS, 141, 193

Davies, R., Mark, D., \& Sternberg, A. 2012, A\&A, 537, A133

Gao, Y., \& Solomon, P. M. 2004a, ApJS, 152, 63

Gao, Y., \& Solomon, P. M. 2004b, ApJ, 606, 271

Gao, Y., Carilli, C. L., Solomon, P. M., \& Vanden Bout P. A. 2007, ApJ, 660, L93

Graciá-Carpio, J., García-Burillo, S., Planesas, P., \& Colina, L. 2006, ApJ, 640, L135

Graciá-Carpio, J., García-Burillo, S., Planesas, P., Fuente, A., \& Usero, A. 2008, A\&A, 479, 703

Greve, T. R., Papadopoulos, P. P., Gao, Y., \& Radford, S. J. E. 2009, ApJ, 692, 1432

Hailey-Dunsheath, S., Sturm, E., Fischer, J., et al. 2012, ApJ, 755, 57

Han, X. H., Zhou, J. J., Wang, J. Z., et al. 2015, A\&A, 576, A131

Hancock, M., Weistrop, D., Nelson, C. H., \& Kaiser, M. E. 2006, AJ, 131, 282

Hernquist, L., \& Mihos, J. C. 1995, ApJ, 448, 41

Kaaret, P., \& Alonso-Herrero, A. 2008, ApJ, 682, 1020

Kaviraj, S. 2014, MNRAS, 440, 2944
Kaviraj, S. 2016, in Galaxies at High Redshift and Their Evolution over Cosmic Time, ed. S. Kaviraj, IAU Symp., 319, 130

Kaviraj, S., Peirani, S., Khochfar, S., Silk, J., \& Kay, S. 2009, MNRAS, 394, 1713

Kennicutt, Jr., R. C. 1998, ApJ, 498, 541

Kohno, K., Kawabe, R., Tosaki, T., \& Okumura, S. K. 1996, ApJ, 461, L29

Kohno, K., Nakanishi, K., Tosaki, T., et al. 2008, Ap\&SS, 313, 279

König, S., Aalto, S., Lindroos, L., et al. 2014, A\&A, 569, A6

Kormendy, J., \& Cornell, M. E. 2004, in Penetrating Bars Through Masks of Cosmic Dust, eds. D. L. Block, I. Puerari, K. C. Freeman, R. Groess, \& E. K. Block, Astrophys. Space Sci. Lib., 319, 261

Krips, M., Neri, R., García-Burillo, S., et al. 2007, A\&A, 468, L63

Krips, M., Neri, R., García-Burillo, S., et al. 2008, ApJ, 677, 262

Krips, M., Martín, S., Eckart, A., et al. 2011, ApJ, 736, 37

Lahuis, F., Spoon, H. W. W., Tielens, A. G. G. M., et al. 2007, ApJ, 659, 296

Lehmer, B. D., Alexander, D. M., Bauer, F. E., et al. 2010, ApJ, 724, 559

Lizano, S., \& Shu, F. H. 1987, in NATO ASIC Proc.210: Physical Processes in Interstellar Clouds, eds. G. E. Morfill, \& M. Scholer, 173

Lotz, J. M., Jonsson, P., Cox, T. J., et al. 2011, ApJ, 742, 103

Matsushita, S., Trung, D.-V., Boone, F., et al. 2015, ApJ, 799, 26

Meier, D. S., Turner, J. L., \& Beck, S. C. 2002, AJ, 124, 877

Meijerink, R., Spaans, M., \& Israel, F. P. 2006, ApJ, 650, L103

Meijerink, R., Spaans, M., \& Israel, F. P. 2007, A\&A, 461, 793

Meijerink, R., Kristensen, L. E., Weiß, A., et al. 2013, ApJ, 762, L16

Murphy, E. J., Condon, J. J., Schinnerer, E., et al. 2011, ApJ, 737, 67

Nguyen Luong, Q., Motte, F., Schuller, F., et al. 2011, A\&A, 529, A41

Papadopoulos, P. P., van der Werf, P. P., Xilouris, E. M., et al. 2012, MNRAS, 426, 2601

Pellerin, A., \& Robert, C. 2007, MNRAS, 381, 228

Pirogov, L. 1999, A\&A, 348, 600

Pozzi, F., Vallini, L., Vignali, C., et al. 2017, MNRAS, 470, L64

Prestwich, A. H., Joseph, R. D., \& Wright, G. S. 1994, ApJ, 422, 73

Privon, G. C., Herrero-Illana, R., Evans, A. S., et al. 2015, ApJ, 814, 39

Sandage, A. 1990, J. R. Astron. Soc. Can., 84, 70

Sandage, A. 2005, ARA\&A, 43, 581

Schmidt, M. 1959, ApJ, 129, 243

Schober, J., Schleicher, D. R. G., \& Klessen, R. S. 2017, MNRAS, 468, 946

Shlosman, I. 2013, Cosmological Evolution of Galaxies, eds. J. Falcón-Barroso, \& J. H. Knapen (Cambridge, UK: Cambridge University Press), 555

Sliwa, K., \& Downes, D. 2017, A\&A, 604, A2

Solomon, P. M., Downes, D., \& Radford, S. J. E. 1992, ApJ, 387, L55

Solomon, P. M., Downes, D., Radford, S. J. E., \& Barrett, J. W. 1997, ApJ, 478, 144

Turner, J. L., Beck, S. C., Benford, D. J., et al. 2015, Nature, 519, 331

Usero, A., García-Burillo, S., Fuente, A., Martín-Pintado, J., \& Rodríguez-Fernández, N. J. 2004, A\&A, 419, 897

Usero, A., Leroy, A. K., Walter, F., et al. 2015, AJ, 150, 115

van der Tak, F. F. S., Black, J. H., Schöier, F. L., Jansen, D. J., \& van Dishoeck E. F. 2007, A\&A, 468, 627

van Haarlem, M. P., Wise, M. W., Gunst, A. W., et al. 2013, A\&A, 556, A2

Weistrop, D., Eggers, D., Hancock, M., et al. 2004, AJ, 127, 1360

Whitmore, B. C. 2007, in Triggered Star Formation in a Turbulent ISM, eds. B. G., Elmegreen, \& J., Palous, IAU Symp., 237, 222

Wynn-Williams, C. G., \& Becklin, E. E. 1993, ApJ, 412, 535 
Appendix A: WideX spectrum of the NOEMA observations

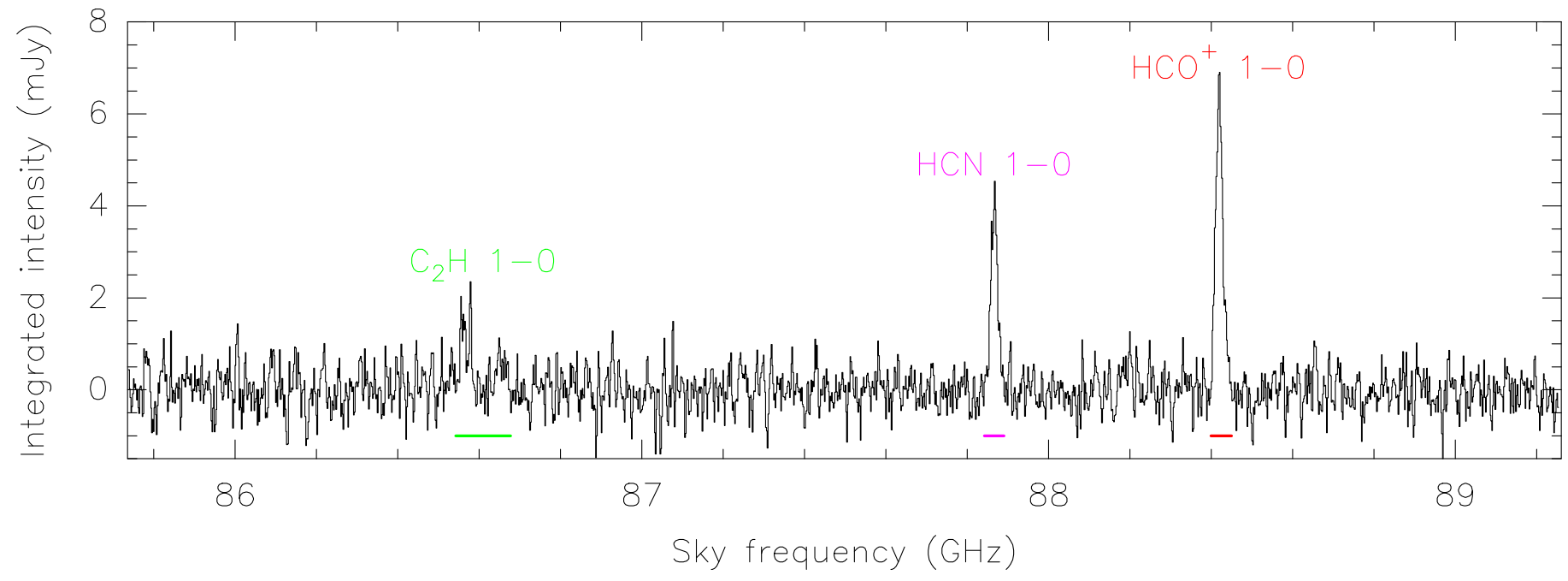

Fig. A.1. NOEMA spectrum of the WideX $3.6 \mathrm{GHz}$ bandwidth (at $\sim 1.95 \mathrm{MHz}\left(\sim 6.6 \mathrm{~km} \mathrm{~s}^{-1}\right)$ channel spacing) extracted from the naturally weighted data cube. The position of the $\mathrm{HCO}^{+}, \mathrm{HCN}$ and $\mathrm{C}_{2} \mathrm{H} 1-0$ lines are indicated in color. We note that due to hyperfine splitting, $\mathrm{C}_{2} \mathrm{H} 1-0$ shows two emission line components.

\section{Appendix B: Comparison of the $3 \mathrm{~mm}$ and $1 \mathrm{~mm}$ continuum images at similar resolution}
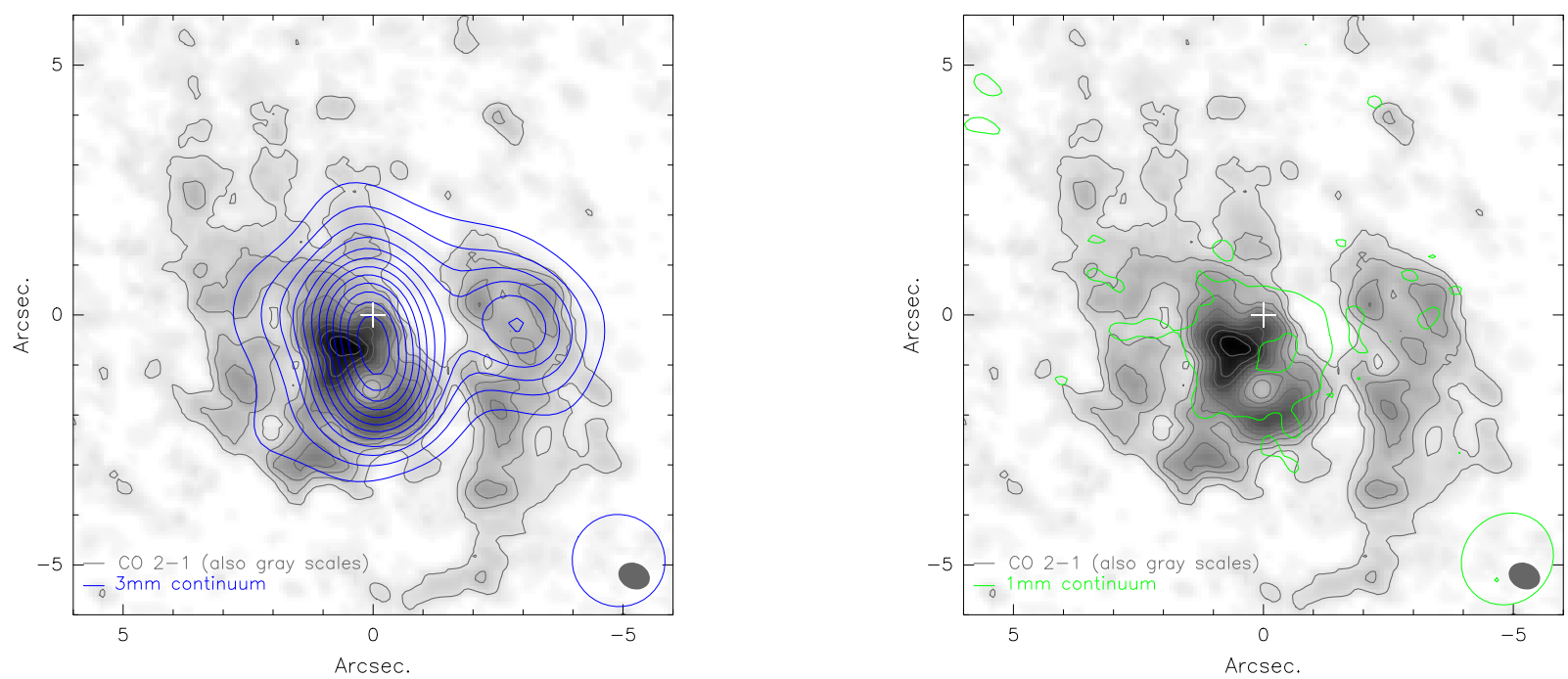

Fig. B.1. $3 \mathrm{~mm}$ (left panel, blue contours) and $1 \mathrm{~mm}$ continuum emission (right panel, green contours) at similar spatial resolution on top of the CO $2-1$ emission (gray contours and background). The $3 \mathrm{~mm}$ continuum contours start at $5 \sigma$ and are spaced in steps of $5 \sigma\left(1 \sigma \sim 31 \mu \mathrm{Jy} \mathrm{beam}{ }^{-1}\right)$. Contours for the $1 \mathrm{~mm}$ continuum emission are at 3 and $6 \sigma\left(1 \sigma \sim 0.5 \mathrm{mJy}_{\text {beam }}^{-1}\right)$. The main continuum emission peak is located south of the AGN position, close to the center of the Eye. However, the $3 \mathrm{~mm}$ continuum peak emission appears elongated; the $1 \mathrm{~mm}$ continuum is of more spherical shape. Also, whereas the $3 \mathrm{~mm}$ continuum shows a secondary emission peak in the western arm, the 1 mm continuum does not. North is up, east to the left. The beam sizes are $1.9^{\prime \prime} \times 1.8^{\prime \prime}$ for both images. 